\title{
Community Generated VR Painting using Eye Gaze
}

\author{
$\mathrm{Mu} \mathrm{Mu}$ \\ mu.mu@northampton.ac.uk \\ University of Northampton \\ Northampton, United Kingdom
}

\author{
Murtada Dohan \\ murtada.dohan@northampton.ac.uk \\ University of Northampton \\ Northampton, United Kingdom
}

\begin{abstract}
This demo introduces an eye-gaze based generative art prototype which was developed to extend the visitors' experience from being audiences to active co-creators for virtual reality (VR) art exhibitions. The design generates a live community artwork based on visitors' visual interactions with VR exhibitions. In a VR exhibition at a public gallery, over 100 visitors participated the new creative process for astonishing community generated artworks.
\end{abstract}

\section{CCS CONCEPTS}

- Human-centered computing $\rightarrow$ Human computer interaction (HCI); • Applied computing $\rightarrow$ Arts and humanities.

\section{KEYWORDS}

eye gaze; virtual reality; abstract art; generative content

\section{ACM Reference Format:}

$\mathrm{Mu} \mathrm{Mu}$ and Murtada Dohan. 2021. Community Generated VR Painting using Eye Gaze. In Proceedings of the 29th ACM International Conference on Multimedia (MM '21), October 20-24, 2021, Virtual Event, China. ACM, New York, NY, USA, 3 pages. https://doi.org/10.1145/3474085.3478552

\section{INTRODUCTION}

With the increasing accessibility of virtual reality (VR) creative tools such as Google Tilt Brush and Masterpiece VR, fully immersive VR art creation and installations are becoming popular in art communities [1][2]. Cooperating with other methodologies, VR painting offers its creators the ultimate ideation platform that supports scalar prototyping to artists as part of a 3D workflow [3] Museums and galleries such as Louvre, Smithsonian Institute, and TATE Modern have been at the vanguard for VR. The transition from physical paint brush and canvas to $3 \mathrm{D}$ virtual environment brings new opportunities as well as challenges. Wearing a VR headset, each visitor can explore artworks on their own in a private virtual exhibition. Despite all benefits, such individual art encounters fall short of the rich visitor interactions and social experience of conventional art exhibitions.

In one of our previous VR user experiments [4], participants were asked to wear a VR headset and walk around to explore a VR abstract art installation. We used a large display unit to show the participant's live headset view to bystanders. This public display

Permission to make digital or hard copies of part or all of this work for personal or classroom use is granted without fee provided that copies are not made or distributed for profit or commercial advantage and that copies bear this notice and the full citation on the first page. Copyrights for third-party components of this work must be honored.

For all other uses, contact the owner/author(s).

MM '21, October 20-24, 2021, Virtual Event, China

(c) 2021 Copyright held by the owner/author(s).

ACM ISBN 978-1-4503-8651-7/21/10

https://doi.org/10.1145/3474085.3478552
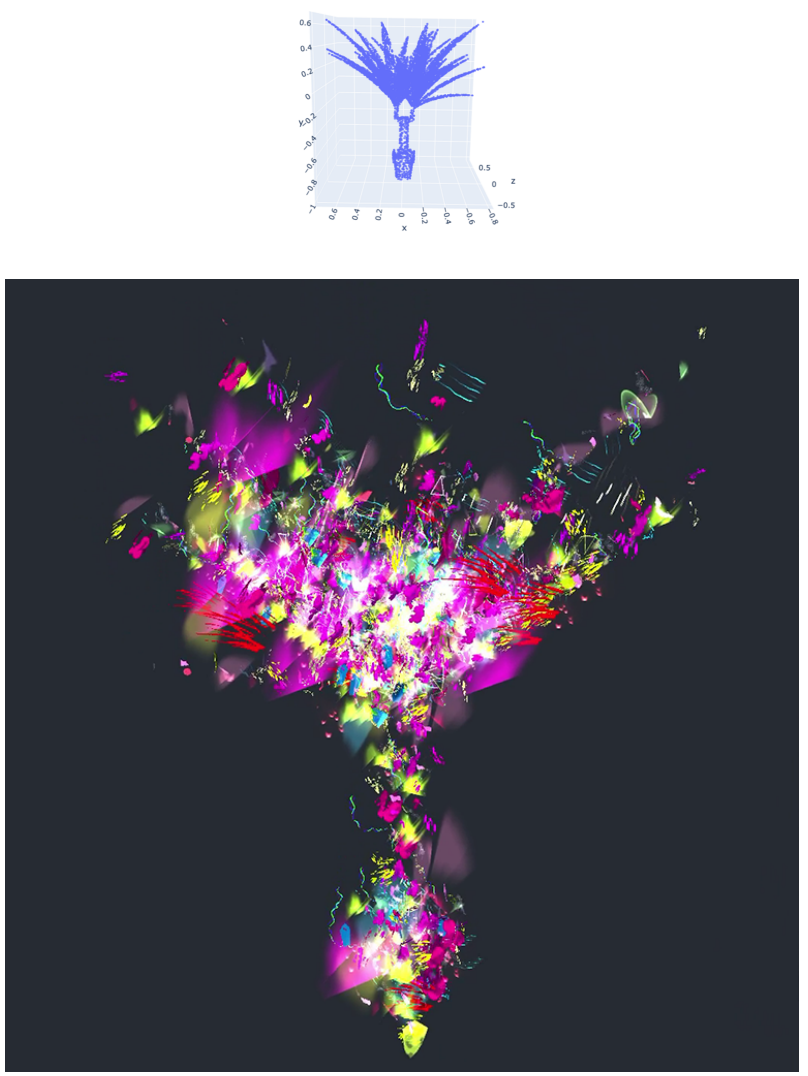

Figure 1: Eye-gaze generated VR artwork

became a catalyst for vibrant interactions between visitors. People were discussing details of the artwork and exchanging ideas of where they will explore. Although our VR art installation was originally designed for individual art encounter, the public display greatly broadened the visitor experience. Post experiment interviews suggested that the interactions with other visitors was a highlight of the overall experience. Such observations led us to the design and experimentation of eye gaze based community generative VR content that can link the personal VR experience with community interactions in the physical space. The unique design allows visitors to become part of the exhibition with no added cognitive load during VR exploration.

\section{SYSTEM OVERVIEW}

The system aims at generating live community content based on how visitors engage with the VR paintings during an exhibition. 


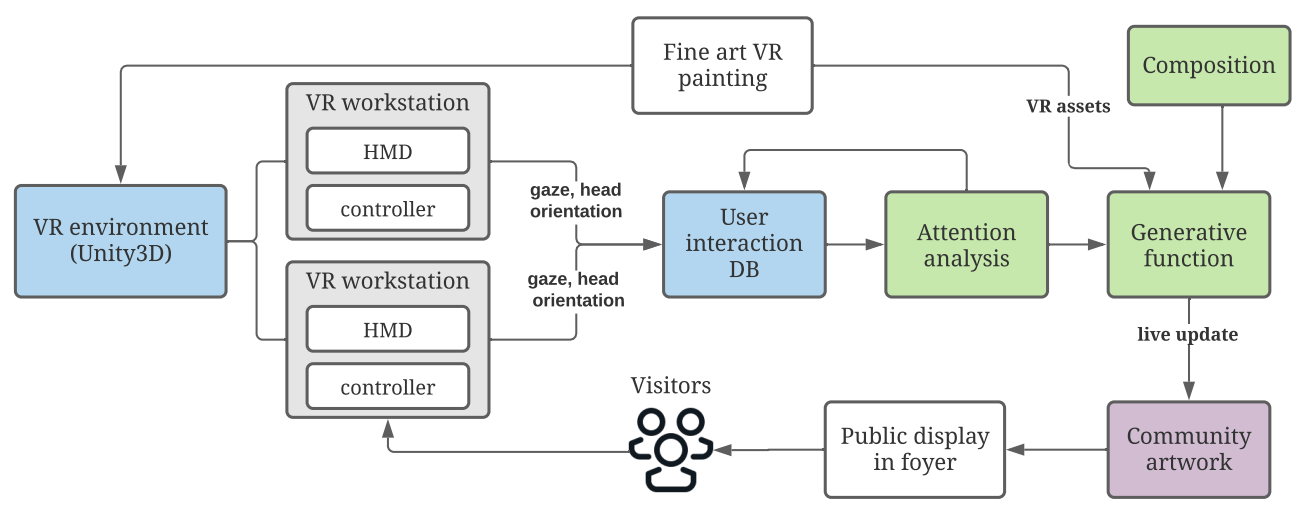

Figure 2: System for Community Generated VR Painting

Each visitor's most favourite parts of the paintings are added to a generated artwork as part of the exhibition. We use eye gaze on brush strokes of VR painting to analyse user interactions with artworks. Compared with other methods such as survey and operations using VR controllers, eye gaze detection is direct, objective and non-intrusive for visual art encounters. Visitor can engage with the artwork as they normally do without explicitly reporting the parts of the artwork that attract their attention.

As depicted in Figure 2, the system contains key elements for VR art installation, tracking, analysis and composition. VR artworks were created by artists using Google Tilt Brush and subsequently imported in a Unity3D-driven VR exhibition environment. Brush strokes of VR paintings are maintained as independent 3D objects for accurate gaze mapping and user attention analysis. A typical abstract VR painting may contain over 10,000 brush strokes in total. The system supports concurrent viewing sessions from multiple VR exhibition stations. Each station is equipped with an eye-tracking enabled VR headset such as HTC VIVE PRO Eye, a controller for VR navigation, and custom Unity3D functions that process raw tracking data (e.g., head and eye orientation). All VR stations push their live tracking data to a common user interaction database.

When a visitor takes off the VR headset at the end of a viewing session, the headset's proximity sensor triggers the attention analysis function to analyse the captured user activities data such as eye-gaze, eye blink, and body and head movements in physical and VR space. The top 30 most popular brush strokes from each viewer are identified with the help of machine learning and the corresponding VR assets are extracted from the original VR artwork for content creation. The generative function maintains the community artwork and appends new content using a selected composition, which determines how VR assets are arranged together. Depending on the preference of an art exhibition, a composition could be:

- A point cloud with discrete 3D coordinates. VR assets are assigned to the coordinates randomly or in a particular order.

- A artist-drawn or equation-defined path or surface. 3D coordinates are sampled on the path or surface.

- A self composition function that mimic the style of a given artwork.
The generative function may keep all original properties of VR assets or apply necessary adjustments. For instance, exhibitors can normalise the size of VR assets so they are equally represented in the result.

\section{PAINT PARK VR EXHIBITION}

Our system was used in a public exhibition Paint Park at MK Gallery in Milton Keynes, UK. Paint Park [5] is an installation of immersive virtual and physical paintings that blurs the lines and boundaries of traditional mark-making through the immersive qualities of virtual reality abstract painting. Visitors were invited to put on VR headsets and explore the VR abstract painting Topsy Turvy. The painting includes two parts. Topsy contains over 40,000 conventional style brush strokes such as Oil Paint and Wet Paint, while Turvey includes over 10,000 special effect brush strokes including Neon Pulse, Chromatic Wave and Electricity.

Figure 1 shows a community generated artwork based on the eye gaze attention of $100+$ visitors. A 3D point cloud of a house plant is used for composition. The resultant VR painting preserves all properties (such as animated light effects) of brushes originally set by the artist. The generated artworks were in display in gallery foyer throughout the public exhibition. Each visitor was able to see how their personal experience was reflected on the artwork immediately after their viewing session. The system supports accelerated replays of the generative process as illustrated in our supplementary submissions. Exhibitors can change the composition on-the-fly.

\section{CONCLUSIONS}

Eye gaze driven community generative art can greatly improve the engagement and social experience of visitors at VR art exhibitions. It also provides artists with a new avenue for creative practices.

At demo sessions of the conference, we plan to demonstrate a live system with the installation of a VR fine art painting. While visitors explore the artwork, they'll be contributing to a ACM MM community generated artwork which will be on display throughout the demo sessions and hosted on a public website for wider audience. The demo will foster discussions on VR system engineering, interactive art practice, generative $\mathrm{AI}$ and human-centred designs. 


\section{REFERENCES}

[1] Yan, Jing, and Mengyu Chen. Reincarnation: virtual reality recreation of Yves Tanguy's world. ACM SIGGRAPH 2019 Virtual, Augmented, and Mixed Reality. 2019.

[2] Jeon, Myounghoon, et al. From rituals to magic: Interactive art and HCI of the past, present, and future. International Journal of Human-Computer Studies 131 (2019)

[3] Herman, Laura M., and Stefanie Hutka. Virtual Artistry: Virtual Reality Translation of Two-Dimensional Creativity. Proceedings of the 2019 on Creativity and Cognition.
2019.

[4] Mu Mu, Murtada Dohan, Alison Goodyear, Gary Hill, Cleyon Johns, Andreas Mauthe. User Attention and Behaviour in Virtual Reality Art Encounter. In submission https://arxiv.org/abs/2005.10161

[5] Paint Park: An experimental installation of immersive virtual and physical abstract paintings by visual artist Alison Goodyear,

https://www.mkgallery.org/whats-on/paint-park/ 